\title{
Estudo Viscosimétrico de Copolímeros à Base de 1,3-Butadieno e 1-Octeno em Tolueno e Hexano*
}

\author{
Gustavo M. Silva, Ivana L. M. Ferreira, Marcia C. Delpech, Marcos A. S. Costa \\ Laboratório de Química de Polímeros, Instituto de Química, Universidade do \\ Estado do Rio de Janeiro (UERJ)
}

\begin{abstract}
Resumo: A viscosimetria é um procedimento experimental simples e pouco oneroso, que pode fornecer informações valiosas sobre o volume hidrodinâmico e a conformação de macromoléculas em solução, num determinado solvente, em uma dada temperatura. Os parâmetros viscosimétricos podem ser matematicamente calculados por extrapolação gráfica, cuja execução experimental é mais demorada. Em contrapartida, é possível que a determinação seja feita por um único ponto. Foi realizado um estudo viscosimétrico, em solução, de polibutadieno e de copolímeros aleatórios de 1,3-butadieno e 1-octeno, (obtidos com diferentes razões, em massa, de 1,3-butadieno/1-octeno $(\mathrm{Bd} / \mathrm{Oct}=99 / 1 \mathrm{e}$ $97 / 3$ ) utilizando-se tolueno e hexano, a $30 \pm 0,1^{\circ} \mathrm{C}$. A massa molar das amostras foi determinada por cromatografia de exclusão por tamanho (SEC). Os cálculos por extrapolação gráfica foram feitos empregando-se as equações de Huggins, Kraemer e Schulz-Blaschke e a determinação por único ponto pelas equações de Schulz-Blaschke, Solomon-Ciuta e Deb-Chanterjee. O método de determinação por um único ponto mostrou-se eficiente para os sistemas estudados. Avaliou-se a qualidade dos solventes por meio da determinação das constantes viscosimétricas de Huggins e Kraemer. Ambos os solventes utilizados podem ser classificados como bons para os polímeros analisados $\left(\mathrm{k}_{\mathrm{h}}<0,5\right.$ e $\left.\mathrm{k}_{\mathrm{k}}<0\right)$, sendo o hexano o melhor, uma vez que as viscosidades intrínsecas determinadas para todas as amostras neste solvente foram maiores do que as determinadas em tolueno. Por meio dos valores de viscosidade intrínseca e do parâmetro g' (razão entre as viscosidades do polímero ramificado e do linear) foi confirmada a existência de ramificações na cadeia polimérica já que g'<1 e os valores de viscosidade intrínseca são inferiores aos obtidos para o homopolímero.
\end{abstract}

Palavras-chave: Copolimerização, 1,3-butadieno, 1-octeno, viscosimetria, determinação por um único ponto.

\section{Viscosimetric Study of Copolymer Based on 1,3-Butadiene and 1-Octene in Toluene and Hexane}

\begin{abstract}
Viscosimetry is a simple, inexpensive characterization method that provides valuable information about the hydrodynamic volume and conformation of macromolecules in solution, in a solvent, at a given temperature. The viscosimetric parameters can be mathematically calculated by graphic extrapolation, in time-consuming experimental tests. It is possible, however, to achieve results by a single point determination. In this work we used the two calculation methods employing a series of six equations: Huggins, Kraemer and Schulz-Blaschke, by graphic extrapolation; and Schulz-Blaschke, Solomon-Ciuta and Deb-Chanterjee, by a single point determination, for solutions of polybutadiene and of random copolymers of 1,3-butadiene and 1-octene, with different mass ratios $(\mathrm{Bd} / \mathrm{Oct}=99 / 1$ and 97/3). The viscosimetric determinations were carried out in toluene and hexane, at $30 \pm 0.1{ }^{\circ} \mathrm{C}$. The weight average molecular mass was determined by size exclusion chromatography (SEC). The values of intrinsic viscosity and of some constants indicated that both toluene and hexane were good solvents for the samples, with hexane being better owing to the higher intrinsic viscosities when compared to the former. It was also observed that the method of calculation by a single point was valid. By means of the intrinsic viscosity values and the parameter g' (ratio between the viscosities of branched and linear polymer) the occurrence of branching in the copolymer chain was suggested because g' $<1$ and the values of intrinsic viscosity were lower than those obtained for the homopolymer.
\end{abstract}

Keywords: Copolymerization, 1,3-butadiene, 1-octene, viscosimetry, one single point determination.

\section{Introdução}

O polibutadieno sintetizado com catalisadores Ziegler-Natta à base de neodímio apresenta elevado teor de unidades 1,4-cis, alta massa molar e alta linearidade. Tais características conferem boas propriedades mecânicas ao polímero e, em contra partida, dificultam sua processabilidade ${ }^{[1-5]}$. Diante disso, uma das possíveis formas de melhorar essa processabilidade é copolimerizar o 1,3-butadieno com alfa-olefinas ${ }^{[6]}$.
Ao se utilizar uma alfa-olefina como comonômero na reação de copolimerização com o 1,3-butadieno, os dois átomos de carbono que formam a ligação dupla da estrutura do alceno são inseridos na cadeia polimérica, ficando os demais átomos como uma ramificação. Assim, ao se empregar o 1-octeno, são geradas ramificações contendo 6 átomos de carbono.

Autor para correspondência: Marcos A. S. Costa, Instituto de Química, Universidade do Estado do Rio de Janeiro - UERJ, Rua São Francisco Xavier, 524, PHLC, sala 310, CEP 20550-900, Rio de Janeiro, RJ, Brasil, e-mail: marcoscosta.iq.uerj@gmail.com *Dedicado à professora Fernanda M. B. Coutinho ${ }^{\dagger}$ que nos deixou durante a realização deste trabalho. 
Em trabalho anterior do nosso grupo ${ }^{[6]}$ foi mostrado que o sistema catalítico empregado na síntese desses copolímeros, não foi capaz de polimerizar a alfa-olefina individualmente, sendo inviável, desta forma, a formação de poli(1-octeno). Diante disso, foi possível garantir que o consumo de alfa-olefina no meio reacional ocorreu somente pela inserção do 1-octeno nas cadeias de polibutadieno, fornecendo ramificações.

A caracterização desses polímeros pode ser feita por diversas técnicas. No entanto, há poucas publicações mencionando medidas viscosimétricas em soluções diluídas, mesmo sendo a viscosimetria uma técnica que fornece resultados importantes.

A análise da viscosidade de uma solução polimérica infinitamente diluída resulta na obtenção de parâmetros relativos à cadeia isolada, tais como a viscosidade intrínseca [ $\eta]$ que pode ser obtida por extrapolação gráfica, à diluição infinita, a partir de diversas equações matemáticas, como: Huggins, Kraemer e Schulz-Blaschke (Equações 1 a 3, respectivamente) ${ }^{[6-12]}$ :

$$
\begin{aligned}
& \eta_{\mathrm{sp}} / \mathrm{c}=[\eta]_{\mathrm{h}+} \mathrm{k}_{\mathrm{h}}[\eta]_{\mathrm{h}}{ }^{2} \mathrm{c} \\
& \ln \eta_{\mathrm{r}} / \mathrm{c}=[\eta]_{\mathrm{k}-} \mathrm{k}_{\mathrm{k}}[\eta]_{\mathrm{k}}{ }^{2} \mathrm{c} \\
& \eta_{\mathrm{sp}} / \mathrm{c}=[\eta]_{\mathrm{sb}}+\mathrm{k}_{\mathrm{sb}}[\eta]_{\mathrm{sb}} \eta_{\mathrm{sp}}
\end{aligned}
$$

onde: $\eta_{\mathrm{r}=}$ viscosidade relativa ou razão de viscosidade $\eta_{\mathrm{sp}}=$ viscosidade específica; $\eta_{\mathrm{sp} / \mathrm{c}=} \eta_{\text {red }}=$ viscosidade reduzida ou número de viscosidade; $[\eta]=\lim \mathrm{c} \rightarrow 0$ $\eta_{\text {red }}=$ viscosidade intrínseca ou número limite de viscosidade; $\mathrm{k}_{\mathrm{h}}, \mathrm{k}_{\mathrm{k}}$ e $\mathrm{k}_{\mathrm{sb}}=$ coeficientes de Huggins, Kraemer e Shulz-Blaschke, respectivamente

Combinando as Equações 1 e 2, e partindo-se da premissa que $\mathrm{k}_{\mathrm{h}}+\mathrm{k}_{\mathrm{k}}=0,5^{[10]}$, Solomon e Ciuta ${ }^{[13]}$ chegaram à Equação 4 para a determinação da viscosidade intrínseca pelo método por um único ponto.

$$
[\eta]=\left[2\left(\eta_{\mathrm{sp}}-\ln \eta_{\mathrm{r}}\right)\right]^{1 / 2} / \mathrm{c}
$$

A Equação 5 foi proposta por Deb e Chantterjee ${ }^{[14]}$ como uma expressão da viscosidade intrínseca, também determinada pelo método de um único ponto.

$$
[\eta]=\left(3 \ln \eta_{\mathrm{r}}+3 / 2 \eta_{\mathrm{sp}}^{2}-3 \eta_{\mathrm{sp}}\right) / \mathrm{c}
$$

Pode-se observar que essas equações são independentes de constantes, ao contrário das Equações 1 a 3, o que permite o cálculo direto, sem a necessidade de extrapolação. A validade desse método, no entanto, deve ser verificada por meio do método tradicional, comparando-se os valores encontrados com os obtidos por um único ponto. Uma análise das diferenças verificadas entre os valores obtidos por extrapolação e diretamente por um único ponto e o erro envolvido na medida, garante mais rapidamente a validade do método, bem como da equação mais adequada. Essa rapidez na análise é de grande interesse quando grandes quantidades de amostras precisam ser analisadas, principalmente em controle de qualidade industrial ${ }^{[7-9,12]}$.

O objetivo geral deste trabalho foi realizar um estudo viscosimétrico, em soluções de tolueno e de hexano, de amostras de copolímeros de 1,3-butadieno (Bd) com 1-octeno (Oct) sintetizados em diferentes razões, em massa, Bd/Oct = 100/0, 99/1 e 97/3 ${ }^{[6]}$. A partir dos resultados obtidos foi avaliada a presença de ramificações nas amostras poliméricas, a qualidade dos solventes e a validade do método de determinação da viscosidade intrínseca por um único ponto para esses copolímeros.

\section{Experimental}

As amostras foram preparadas utilizando-se um sistema catalítico Ziegler-Natta à base de neodímio, de acordo com procedimento descrito em trabalho anterior ${ }^{[6]}$.

A determinação dos parâmetros viscosimétricos foi realizada a $30 \pm 0,1{ }^{\circ} \mathrm{C}$, utilizando-se um viscosímetro capilar Ubbelohde E431-0B em banho termostatizado Thermo Haake DC30. Foram preparadas soluções a $0,5 \%(\mathrm{~m} / \mathrm{v})$ em tolueno e em hexano. A determinação experimental foi feita pela cronometragem do tempo de escoamento dos solventes, da solução-mãe polimérica e de cinco diluições da mesma (para cada diluição foram adicionados $2 \mathrm{~mL}$ de tolueno ou hexano). Nas determinações por um único ponto, escolheu-se a solução de menor concentração para os cálculos ${ }^{[7,11]}$. É importante ressaltar que o tempo de escoamento da solução polimérica não deve ser muito baixo, pois aumenta a probabilidade de haver erros de leitura. Além disso, tempos de escoamento muito altos significam que a solução polimérica apresenta alta concentração, característica esta que não obedece ao conceito de regime newtoniano.

As massas molares foram determinadas por cromatografia de exclusão por tamanho (SEC), utilizando cromatógrafo Waters 600, equipado com injetor automático Waters 717 Autosampler, detector de índice de refração 2410 e colunas de Styragel com limites de exclusão entre 50 e $1 \times 10^{6} \AA$, calibradas com 15 padrões monodispersos de poliestireno, com massas molares variando de $11,6 \times 10^{6}$ a $1,47 \times 10^{3} \mathrm{~g} / \mathrm{mol}$. As medidas foram conduzidas a $30{ }^{\circ} \mathrm{C}$ e as amostras injetadas automaticamente $(1 \mathrm{~mL} / \mathrm{min})$ como soluções a $0,15 \%$ $(\mathrm{m} / \mathrm{v})$ em tetra-hidrofurano (THF).

\section{Resultados e Discussão}

O parâmetro $c[\eta]$ ("dimensionless coil overlap parameter", onde c é a concentração), pode ser definido como o volume total ocupado pelos novelos poliméricos; isto é, c[ๆ] é a medida da fração, em volume, do polímero presente na solução. Esse parâmetro é independente do tipo e da massa molar do polímero ${ }^{[8]}$.

A Figura 1 apresenta a relação entre $\log \eta_{\mathrm{sp}}$ e $\log$ $\mathrm{c}[\eta]_{\mathrm{h}}$ obtida para o homopolímero $(\mathrm{Bd} / \mathrm{Oct}=100 / 0)$ e os copolímeros $(\mathrm{Bd} / \mathrm{Oct}=99 / 1$ e 93/7), nos diferentes solventes analisados (tolueno e hexano), empregandose os valores da viscosidade intrínseca obtida pela equação de Huggins. Foi observada uma relação linear para todas as amostras analisadas, comprovando assim, que todas as determinações foram executadas em regime newtoniano ${ }^{[8,9,12]}$. 
Avaliação da presença de ramificações na cadeia polibutadiência a partir da determinação da viscosidade intrínseca de Huggins $\left[\eta_{h}\right]$ e do parâmetro $g$ '

A literatura ${ }^{[15]}$ descreve que o volume hidrodinâmico de um polímero ramificado é sempre menor do que o volume ocupado por um polímero linear de mesma massa molar, já que as ligações covalentes existentes entre as cadeias do polímero ramificado limitam o seu coeficiente de expansão e, assim, provocam uma diminuição na viscosidade intrínseca [ $\eta$, em virtude de ocorrer a incorporação de ramificações na cadeia polimérica.

A Figura 2 mostra as retas obtidas através da equação de Huggins para o homopolímero $(\mathrm{Bd} /$ Oct $=100 / 0)$ e os copolímeros $(\mathrm{Bd} /$ Oct $=99 / 1$ e 97/3). É possível verificar que os copolímeros, independente da razão utilizada, apresentaram valores de viscosidade intrínseca inferiores ao homopolímero (Tabela 1). Este resultado sugere a possível formação de ramificações nas cadeias polibutadiênicas, em virtude da incorporação da alfa-olefina na cadeia polimérica.

O efeito da ramificação pode ser estudado a partir de medidas de viscosidade intrínseca, realizadas em bons solventes, utilizando-se o parâmetro g' (Equação 6), onde se determina a razão entre as viscosidades do polímero ramificado e do polímero linear ${ }^{[15]}$.

$$
g^{\prime}=\left[\frac{[\eta]_{\text {ramificado }}}{[\eta]_{\text {linear }}}\right]_{M}
$$

Sendo $[\eta]_{\text {ramificado }}$ e $[\eta]_{\text {linear }}$ os valores de viscosidade intrínseca de Huggins para o polímero ramificado e o linear, respectivamente, com massas molares similares.
Quando a solução polimérica é preparada em bons solventes, o coeficiente de expansão é influenciado pela ramificação. Um polímero linear é capaz de se expandir muito mais do que um polímero ramificado. Desta forma, os polímeros ramificados possuem dimensões menores do que os polímeros lineares, originando assim, um valor de g' sempre menor do que a unidade ${ }^{[15]}$.

Analisando-se os resultados apresentados na Tabela 2, pode-se notar que os valores de g' confirmam a presença de ramificações nas amostras copolimerizadas $(\mathrm{Bd} / \mathrm{Oct}=99 / 1$ e 97/3), já que tais valores foram menores do que a unidade e as massas molares ponderais médias $\left(\mathrm{M}_{\mathrm{w}}\right)$, obtidas por SEC, dos copolímeros apresentam valores próximos à massa obtida para o homopolímero.

Os resultados mostrados nesta etapa do trabalho permitem sugerir que é possível avaliar a presença de ramificações em cadeias poliméricas a partir de medidas viscosimétricas realizadas em solução.

\section{Avaliação da qualidade do solvente para os sistemas estudados}

A fim de se avaliar a qualidade dos solventes utilizados neste trabalho e assim confirmar a veracidade dos valores calculados para o parâmetro g', foram calculadas as constantes viscosimétricas de Huggins $\left(\mathrm{k}_{\mathrm{h}}\right)$ e de Kraemer $\left(\mathrm{k}_{\mathrm{k}}\right)$ (Tabela 3).

A Tabela 3 mostra as constantes viscosimétricas obtidas para os polímeros. A constante de Huggins $\left(\mathrm{k}_{\mathrm{h}}\right)$ fornece informações a respeito da interação polímerosolvente, indicando que quanto menor for o seu valor, melhor será o solvente, isto é, mais estendida estará a

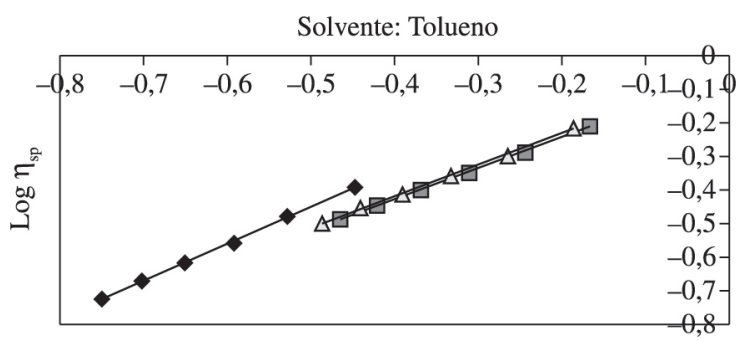

$\log c[\eta]_{H}$

- $\mathrm{Bd} / \mathrm{Oct}=100 / 0 \quad \square \mathrm{Bd} / \mathrm{Oct}=99 / 1 \quad \Delta \mathrm{Bd} / \mathrm{Oct}=97 / 3$

(a)

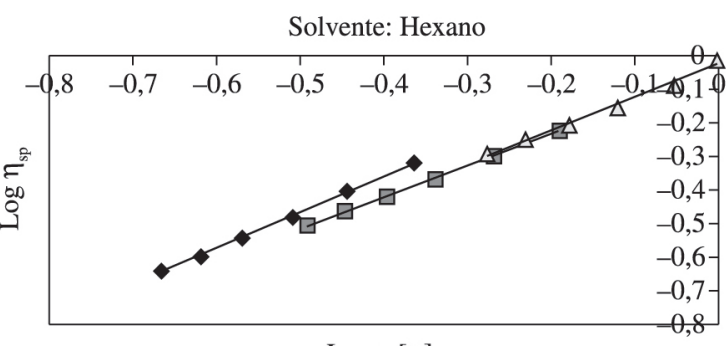

$\log c[\eta]_{\mathrm{H}}$

- $\mathrm{Bd} / \mathrm{Oct}=100 / 0 \quad \square \mathrm{Bd} / \mathrm{Oct}=99 / 1 \quad \Delta \mathrm{Bd} / \mathrm{Oct}=97 / 3$

(b)

Figura 1. $\log \eta_{\mathrm{sp}}$ versus $\log \mathrm{c}[\eta]_{\mathrm{H}}$ para todas as amostras em: (a) tolueno e (b) hexano.

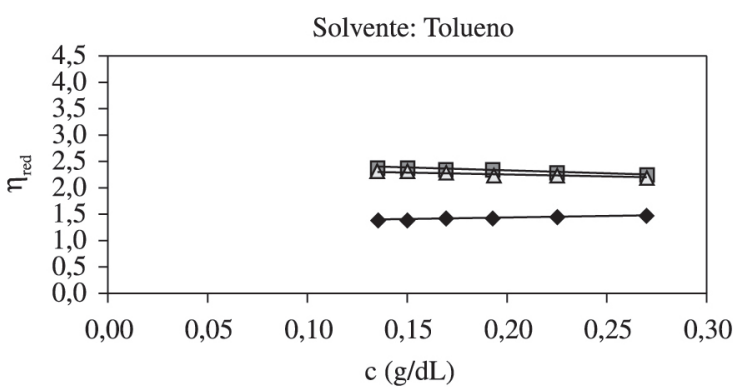

- $\mathrm{Bd} /$ Oct $=100 / 0 \square \mathrm{Bd} /$ Oct $=99 / 1 \Delta \mathrm{Bd} /$ Oct $=97 / 3$

(a)

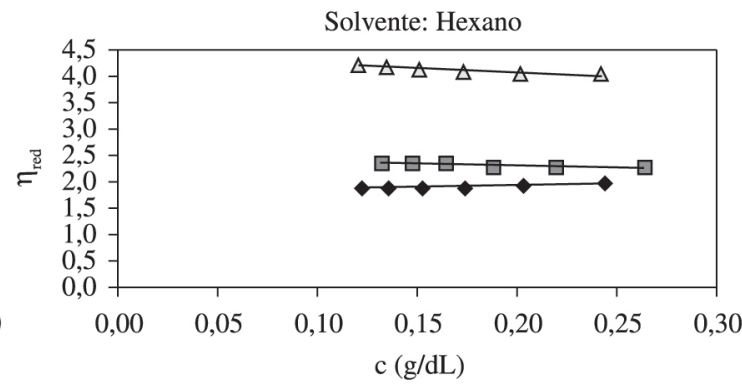

- Bd/Oct $=100 / 0 \square \mathrm{Bd} /$ Oct $=99 / 1 \Delta \mathrm{Bd} /$ Oct $=97 / 3$

(b)

Figura 2. Determinação da viscosidade intrínseca de Huggins para todas as amostras em: (a) tolueno e (b) hexano. 
Tabela 1. Valores de viscosidade intrínseca para o homopolímero $(\mathrm{Bd} / \mathrm{Oct}=100 / 0)$ e os copolímeros $(\mathrm{Bd} / \mathrm{Oct}=99 / 1$ e 97/3) em tolueno e em hexano, a $30{ }^{\circ} \mathrm{C}$.

\begin{tabular}{|c|c|c|c|c|c|c|}
\hline \multicolumn{7}{|c|}{ TOLUENO } \\
\hline \multirow{3}{*}{ Amostra } & \multicolumn{6}{|c|}{ Viscosidade intrínseca [ $\eta]$ (dL/g) } \\
\hline & \multicolumn{3}{|c|}{ Extrapolação gráfica } & \multicolumn{3}{|c|}{ Único ponto } \\
\hline & $\mathbf{H}^{(\mathrm{a})}$ & $\mathbf{K}^{(\mathbf{a})}$ & $\mathbf{S B}^{\text {(a) }}$ & $\mathbf{S B}^{(\mathbf{b})}$ & $\mathbf{S C}^{(\mathrm{c})}$ & DC $^{(\mathrm{c})}$ \\
\hline $\mathrm{Bd} / \mathrm{Oct}=100 / 0^{(\mathrm{d})}$ & 3,348 & 3,523 & 3,982 & 3,643 & 3,716 & 4,027 \\
\hline $\mathrm{Bd} / \mathrm{Oct}=99 / 1$ & 2,435 & 2,563 & 2,896 & 2,650 & 2,702 & 2,929 \\
\hline $\mathrm{Bd} / \mathrm{Oct}=97 / 3$ & 2,865 & 3,015 & 3,408 & 3,118 & 3,180 & 3,446 \\
\hline \multicolumn{7}{|c|}{ HEXANO } \\
\hline \multirow{3}{*}{ Amostra } & \multicolumn{6}{|c|}{ Viscosidade intrínseca [ ]] (dL/g) } \\
\hline & \multicolumn{3}{|c|}{ Extrapolação gráfica } & \multicolumn{3}{|c|}{ Único ponto } \\
\hline & $\mathbf{H}^{(\mathrm{a})}$ & $\mathbf{K}^{(\mathbf{a})}$ & $\mathbf{S B}^{(\mathbf{a})}$ & $\mathbf{S B}^{(\mathbf{b})}$ & $\mathbf{S C}^{(\mathrm{c})}$ & $\mathbf{D C}^{(\mathrm{c})}$ \\
\hline $\mathrm{Bd} / \mathrm{Oct}=100 / 0^{(\mathrm{d})}$ & 4,390 & 4,383 & 4,562 & 4,451 & 4,412 & 4,580 \\
\hline Bd/Oct (99/1) & 3,557 & 3,527 & 3,739 & 3,479 & 3,453 & 3,504 \\
\hline Bd/Oct (97/3) & 3,145 & 3,223 & 3,504 & 3,321 & 3,318 & 3,060 \\
\hline
\end{tabular}

${ }^{(a)} \mathrm{H}=$ Huggins; $\mathrm{K}=$ Kraemer; $\mathrm{SB}=$ Schulz-Blascke; ${ }^{(\mathrm{b})} \mathrm{SB}=$ Schulz-Blascke $\left(\mathrm{k}_{\mathrm{sb}}=0,28\right) ;{ }^{\left({ }^{c}\right)} \mathrm{SC}=$ Solomon-Ciuta; DC = Deb-Chanterjee;

(d) Homopolímero (polibutadieno alto-cis).

Tabela 2. Valores de massa molar ponderal média (Mw) e do parâmetro g'.

\begin{tabular}{|c|c|c|c|c|c|}
\hline \multirow{2}{*}{ Amostra } & \multirow{2}{*}{$\operatorname{Mw}\left(\mathbf{x 1 0 ^ { - 5 }}\right)^{(\mathrm{a})}$} & \multicolumn{2}{|c|}{ TOLUENO } & \multicolumn{2}{|c|}{ HEXANO } \\
\hline & & {$[\eta]_{h}(\mathbf{d L} / g)^{(\mathbf{b})}$} & g' & {$[\eta]_{h}(\mathbf{d L} / \mathbf{g})^{(\mathbf{b})}$} & g' \\
\hline $\mathrm{Bd} / \mathrm{Oct}=100 / 0^{(\mathrm{c})}$ & 3,3 & 3,348 & - & 4,390 & - \\
\hline $\mathrm{Bd} / \mathrm{Oct}=99 / 1$ & 3,0 & 2,435 & 0,73 & 3,557 & 0,81 \\
\hline $\mathrm{Bd} / \mathrm{Oct}=97 / 3$ & 3,5 & 2,865 & 0,86 & 3,145 & 0,72 \\
\hline
\end{tabular}

(a) Massa molar ponderal média determinada por SEC, em THF; (b)Viscosidade intrínseca de Huggins; ${ }^{(\mathrm{c})}$ Homopolímero (polibutadieno alto-cis).

Tabela 3. Constantes viscosimétricas determinadas para o homopolímero $(\mathrm{Bd} / \mathrm{Oct}=100 / 0)$ e os copolímeros $(\mathrm{Bd} / \mathrm{Oct}=99 / 1$ e $97 / 3)$ em tolueno e hexano, a $30^{\circ} \mathrm{C}$.

\begin{tabular}{|c|c|c|c|}
\hline \multicolumn{4}{|c|}{ TOLUENO } \\
\hline \multirow{2}{*}{ Amostra } & \multicolumn{3}{|c|}{ Constantes viscosimétricas } \\
\hline & $\mathbf{k}_{\mathrm{h}}^{(\mathrm{a})}$ & $\mathbf{k}_{\mathrm{k}}^{(\mathrm{a})}$ & $\mathbf{k}_{h}+\mathbf{k}_{\mathrm{k}}$ \\
\hline $\mathrm{Bd} / \mathrm{Oct}=100 / 0^{(\mathrm{b})}$ & 0,508 & $-0,113$ & 0,395 \\
\hline $\mathrm{Bd} / \mathrm{Oct}=99 / 1$ & 0,422 & $-0,082$ & 0,340 \\
\hline $\mathrm{Bd} / \mathrm{Oct}=97 / 3$ & 0,499 & $-0,097$ & 0,402 \\
\hline \multicolumn{4}{|l|}{ HEXANO } \\
\hline \multirow{2}{*}{ Amostra } & \multicolumn{3}{|c|}{ Constantes viscosimétricas } \\
\hline & $\mathbf{k}_{\mathrm{h}}^{(\mathrm{a})}$ & $\mathbf{k}_{\mathrm{k}}^{(\mathrm{a})}$ & $k_{h}+k_{k}$ \\
\hline $\mathrm{Bd} / \mathrm{Oct}=100 / 0^{(\mathrm{b})}$ & 0,351 & $-0,136$ & 0,215 \\
\hline $\mathrm{Bd} /$ Oct $=99 / 1$ & 0,410 & $-0,145$ & 0,265 \\
\hline $\mathrm{Bd} / \mathrm{Oct}=97 / 3$ & 0,473 & $-0,126$ & 0,347 \\
\hline
\end{tabular}

${ }^{(\mathrm{a})} \mathrm{h}=$ Huggins; $\mathrm{k}=$ Kraemer; ${ }^{\left({ }^{b}\right)}$ Homopolímero (polibutadieno alto-cis).

macromolécula $^{[16]}$. Dados experimentais mostram que valores menores que 0,5 são encontrados para soluções poliméricas diluídas em um bom solvente. Assim, a partir dos resultados apresentados na Tabela 3 é possível afirmar que, independente do teor de 1-octeno contido na amostra, os solventes utilizados (tolueno e hexano) podem ser considerados bons solventes para os polímeros analisados neste trabalho. Tal fato é confirmado através dos valores negativos encontrados para a constante de $\operatorname{Kraemer}\left(\mathrm{k}_{\mathrm{k}}\right)$. A $k_{k}$ depende da razão entre o volume hidrodinâmico ocupado por duas moléculas de soluto que interagem entre si e o volume hidrodinâmico ocupado por uma molécula isolada. Quando o seu valor é negativo significa que há uma boa solubilização da cadeia polimérica, ou seja, há maior interação polímero-solvente ${ }^{[7-12]}$.

\section{Avaliação do método de determinação da viscosidade intrínseca por um único ponto}

Para a determinação por um único ponto, foram empregadas as equações de Solomon-Ciuta (SC) e DebChanterjee (DC) (Equações 4 e 5, respectivamente). Um teste também foi feito, empregando-se a Equação 3, de Shulz-Blasche (SB), neste caso atribuindo o valor de 0,28 à respectiva constante $\left(\mathrm{k}_{\mathrm{SB}}\right)$, uma vez que a literatura mostra este valor sendo constante para um grande número de sistemas polímero/solvente ${ }^{[7-12]}$. Assim, considerando $\mathrm{k}_{\mathrm{SB}}=0,28$ e substituindo-o na Equação 3, tem-se:

$\eta_{\mathrm{sp}} / \mathrm{c}=[\eta]_{\mathrm{sb}}+(0,28)[\eta]_{\mathrm{sb}} \eta_{\mathrm{sp}}(7)$

Existem trabalhos na literatura que utilizam essas equações para a determinação da viscosidade intrínseca ${ }^{[6-12]}$. Entretanto, até o presente momento, não foi encontrada a aplicação de tais equações para copolímeros de 1,3-butadieno e 1-octeno.

$\mathrm{O}$ uso dessas equações tem sido feito a partir da premissa que $\mathrm{k}_{\mathrm{h}+} \mathrm{k}_{\mathrm{k}=} 0,5^{[10]}$. Porém, conforme mostrado na Tabela 3, a relação $\mathrm{k}_{\mathrm{h}}+\mathrm{k}_{\mathrm{k}}$, neste trabalho, variou de 0,215 a 0,402 . No entanto, tanto para os valores de $[\eta]_{\mathrm{SC}}$ quanto os de $[\eta]_{\mathrm{DC}}$, os dados encontrados são próximos àqueles obtidos por extrapolação gráfica $\left([\eta]_{\mathrm{h}},[\eta]_{\mathrm{k}}\right.$ e $\left.[\eta]_{\mathrm{sb}}\right)($ Tabela 1). A Tabela 4 mostra a diferença percentual entre os valores de viscosidade intrínseca obtidos pelas Equações 2 a 5, tendo como base a equação de Huggins (Equação 1), mais comumente empregada em viscosimetria. 
Tabela 4. Diferenças percentuais $(\Delta \%)$ obtidas para valores de viscosidade intrínseca calculados por extrapolação gráfica (Kraemer (K) e Schulz-Blaschke (SB)) e por medidas por um único ponto (Schulz-Blaschke (SB), Solomon-Ciuta (SC) e Deb-Chanterjee (DC)), tomando como referência a viscosidade intrínseca de Huggins, $[\eta]_{\mathrm{h}}$

\begin{tabular}{|c|c|c|c|c|c|}
\hline \multicolumn{6}{|c|}{ Diferença percentual $(\Delta \%)^{(a)}$ para os valores de viscosidade intrínseca } \\
\hline \multirow{3}{*}{ Amostra } & \multicolumn{5}{|c|}{\begin{tabular}{|c|} 
TOLUENO \\
\end{tabular}} \\
\hline & \multicolumn{2}{|c|}{ Extrapolação gráfica } & \multicolumn{3}{|c|}{ Medidas por um único ponto } \\
\hline & $\mathbf{K}$ & SB & SB & SC & DC \\
\hline $\mathrm{Bd} / \mathrm{Oct}=100 / 0^{(\mathrm{b})}$ & 5,23 & 18,94 & 8,81 & 10,99 & 20,28 \\
\hline $\mathrm{Bd} / \mathrm{Oct}=99 / 1$ & 5,23 & 18,93 & 8,83 & 10,97 & 20,29 \\
\hline \multirow[t]{2}{*}{$\mathrm{Bd} / \mathrm{Oct}=97 / 3$} & 5,23 & 18,95 & 8,83 & 10,99 & 20,28 \\
\hline & \multicolumn{5}{|c|}{ HEXANO } \\
\hline \multirow[t]{2}{*}{ Amostra } & \multicolumn{2}{|c|}{ Extrapolação gráfica } & \multicolumn{3}{|c|}{ Medidas por um único ponto } \\
\hline & $\mathbf{K}$ & SB & SB & SC & DC \\
\hline $\mathrm{Bd} / \mathrm{Oct}=100 / 0^{(\mathrm{b})}$ & $-0,16$ & 3,92 & 1,39 & 0,50 & 4,33 \\
\hline $\mathrm{Bd} / \mathrm{Oct}=99 / 1$ & $-0,84$ & 5,12 & $-2,19$ & $-2,92$ & $-1,49$ \\
\hline $\mathrm{Bd} / \mathrm{Oct}=97 / 3$ & 2,48 & 11,41 & 5,60 & 5,50 & $-2,70$ \\
\hline
\end{tabular}

${ }^{(a)} \Delta \%=\left[100\left([\eta] /[\eta]_{\mathrm{h}}\right)\right]-100 ;{ }^{(b)}$ Homopolímero (polibutadieno alto-cis).

As viscosidades intrínsecas obtidas pelo método de determinação por um único ponto empregando-se a equação de Schulz-Blaschke (SB) apresentaram menores diferenças percentuais (aproximadamente $8,8 \%$ ), quando comparadas àquelas obtidas pelo método de extrapolação gráfica usando-se a mesma equação (aproximadamente $18,9 \%$ ) (Tabela 4). Isso confirma que $\mathrm{k}_{\mathrm{SB}}=0,28$ também pode ser empregada para as amostras estudadas neste trabalho. Desse modo, os resultados indicam que, para os sistemas copolímeros de 1,3-butadieno e 1-octeno / tolueno, a equação SB é a mais adequada na determinação por um único ponto.

Para o solvente hexano, a equação SB, aplicada em um único ponto, mostrou diferenças percentuais ainda menores, quando comparada com a extrapolação gráfica. Comprovando assim, que o valor 0,28 também é adequado para os sistemas em hexano.

Comparando-se os resultados obtidos a partir das equações SC e DC para os sistemas estudados, é possível verificar que foram obtidos valores expressivamente menores de diferenças percentuais quando se utilizou a equação de $\mathrm{SC}$ em tolueno. Em hexano, não houve diferença significativa entre as equações, todas conduziram a diferenças percentuais próximas e reduzidas, indicando que ambas podem ser empregadas.

\section{Conclusões}

O método de determinação por um único ponto se mostrou eficiente na determinação da viscosidade intrínseca dos copolímeros, tanto em tolueno como em hexano, a $30^{\circ} \mathrm{C}$. Para as análises realizadas com o tolueno, a equação que se mostrou mais apropriada foi a de SchulzBlaschke. Em hexano, as três equações (Schulz-Blaschke, Solomon-Ciuta e Deb-Chanterjee) foram apropriadas. O valor de 0,28 para a constante de Schulz-Blaschke foi aplicável aos sistemas nos dois solventes.

Tanto o tolueno como o hexano podem ser considerados bons solventes para os copolímeros estudados $\left(\mathrm{k}_{\mathrm{h}}<0,5\right.$ e $\left.\mathrm{k}_{\mathrm{k}}<0\right)$. Porém, o hexano se mostrou melhor solvente, uma vez que apresentou maiores valores de viscosidade intrínseca.
Os valores de g' $(<1)$ indicaram a presença de ramificações na cadeia polimérica.

\section{Agradecimentos}

Os autores agradecem à Fundação Carlos Chagas Filho de Amparo à Pesquisa do Estado do Rio de Janeiro (FAPERJ) pelo suporte financeiro.

\section{Referências Bibliográficas}

1. Mello, I. L.; Coutinho, F. M. B.; Nunes, D. S. S.; Soares, B. G.; Costa, M. A. S. \& Santa-Maria, L. C. - Eur. Polym. J. 40, p.635 (2004). http://dx.doi.org/10.1016/j. eurpolymj.2003.10.023

2. Ferreira, C. N.; Mello, I.L. \& Coutinho, F.M.B. - Polímeros, 19 p.138 (2009). http://dx.doi.org/10.1590/S010414282009000200011

3. Nunes, D. S. S.; Coutinho, F. M. B. \& Mello, I. L. - Polímeros, 15, p.296 (2005).

4. Mello, I. L.; Soares, B. G.; Coutinho, F. M. B. \& Nunes, D. S. S. - Polímeros, 14, p.235 (2004).

5. Pires, N. M. T. - "Estudo de sistemas catalíticos à base de neodímio para a produção de polibutadieno alto-cis”, Tese de Doutorado, Universidade Federal do Rio de Janeiro, Brasil (2004).

6. Silva, G. M.; Coutinho, F. M. B.; Mello, I. L.; Costa, M. A. S. \& Junger, D. F. C. - Polímeros, 21, p.311 (2011). http:// dx.doi.org/10.1590/S0104-14282011005000059

7. Mello, I. L.; Delpech, M. C.; Coutinho, F. M. B.; Albino, F. F. M. - J. Braz. Chem. Soc., 17, p.194 (2006). http://dx.doi. org/10.1590/S0103-50532006000100028

8. Mello, I. L.; Delpech, M. C.; Coutinho, F. M. B.; Albino, F. F. M.; Santos, S. M. - Polímeros, 16, p.53 (2006) http:// dx.doi.org/10.1590/S0104-14282006000100012

9. Mello, I. L.; Delpech, M. C.; Coutinho, F. M. B.; Albino, F. F. M. \& Santos, S. M. - Polym. Bull., 55, p.115 (2005). http://dx.doi.org/10.1007/s00289-005-0420-3

10. Abdel-Azim, A. A. A.; Atta, A. M.; Farahat, M. S.; Boutros, W. Y. - Polymer, 39, p.26 (1998). 
11. Delpech, M. C.; Coutinho, F. M. B.; Sousa, K. G. M.; Cruz, R. C. - Polímeros, 17, p.294 (2007). http://dx.doi. org/10.1590/S0104-14282007000400008

12. Delpech, M. C.; Oliveira, C. M. F. - Polym. Test., 24, p.381 (2005). http://dx.doi.org/10.1016/j. polymertesting.2004.09.012

13. Solomon, O.F.\& Ciuta, I. Z. - J. Appl. Polym. Sci., 6, p.683 (1962) http://dx.doi.org/10.1002/app.1962.070062414

14. Deb, P. C.\& Chantterjee, S. R. - J. Appl. Chem., 31, p.121 (1968).
15. Lucas, E. F.; Soares, B. G.; Monteiro, E. E. - "Caracterização de polímeros: Determinação de peso molecular e análise térmica", E-papers, Rio de Janeiro (2001).

16. Huggins, M. L. - J. Am. Chem. Soc, 64, p.2716 (1942). http://dx.doi.org/10.1021/ja01263a056

Enviado: 20/07/12 Reenviado: 02/11/12 Aceito: $21 / 11 / 12$ 